Conclusion: The results showed that the positive effect of multimodal occupational therapy on pain and hand function persisted over the 2-year period, however, no significant between-groups difference over time was found. No significant between-group differences were found when dividing into sub-groups, however, those later undergoing surgery in the control group scored significantly worse on pain and hand function at the time of surgical consultation. The results may imply that patients who would benefit from surgery were identified, and that surgery does not give an additional benefit in patients who have received multimodal occupational therapy. This needs to be further investigated.

REFERENCES:

[1] Tveter AT, Østerås N, Nossum R, Eide REM, Klokkeide Å, Hoegh Matre $\mathrm{K}$, et al. Short-term effects of occupational therapy on hand function and pain in patients with carpometacarpal osteoarthritis: secondary analyses from a randomized controlled trial. Arthritis care \& research. 2020:10.1002/ acr.24543

Acknowledgements: We would like to acknowledge Øyvor Andreassen for her contribution throughout the project as a patient representative.

Disclosure of Interests: None declared

DOI: 10.1136/annrheumdis-2021-eular.2857

\section{OP0158-HPR THE FALL PREVENTION PROGRAMME 'SICHER DURCH DEN ALLTAG' IS EFFECTIVE AND COST-EFFECTIVE}

$\underline{\text { K. Niedermann Schneider }}{ }^{1}$, A. Meichtry ${ }^{1}$, B. Zindel ${ }^{2}$, B. Brunner ${ }^{3}$, M. Ernst ${ }^{1}$, V. Krafft ${ }^{2}$, R. Mattli ${ }^{3}$, I. Nast ${ }^{1}$, M. Wirz ${ }^{1}$, S. Wieser ${ }^{3}$. ${ }^{1}$ Zurich University of Applied Sciences, Institute of Physiotherapy, Winterthur, Switzerland; ${ }^{2}$ Swiss League Against Rheumatism, Swiss League Against Rheumatism, Zurich, Switzerland: ${ }^{3}$ Zurich University of Applied Sciences, Winterthur Institute of Health Economics, Winterthur, Switzerland

Background: Falls in older people are frequent and have often severe health consequences. Reinforced by population ageing, they represent a substantial challenge for health care systems. Falls are often due to a combination of intrinsic and extrinsic risk factors. For these reasons, the Swiss League Against Rheumatism (SLAR) developed the multidimensional, home-based fall prevention programme 'Sicher durch den Alltag' ('Safely Through Everyday Life'). The programme has been implemented nationwide since 2013 and is financed by selected health insurance companies. Trained physiotherapists (PTs) or occupational therapists (OTs) visit the senior at home for a 60-90 minutes session. They perform a detailed assessment of the senior's individual risk of falling and subsequently eliminate identified environmental risk factors and provide tailored exercises. After 4 weeks, the PT/OT calls the senior to discuss further needs. The cost of the intervention is at 500 Swiss Francs (approximately 460 Euro) per senior.

Objectives: Assess the effectiveness and cost-effectiveness of this fall prevention programme.

Methods: A prospective, longitudinal observational study was performed. Baseline study data was assessed at the home visit, including the self-reported number of falls during $a$ ) the year and $b$ ) the month before the visit. The participants of the programme were monitored for one year after the home visit by bi-monthly telephone calls. Participants were asked to report the falls that they continuously documented in a diary. Questionnaires administered orally included the Falls Efficacy Scale-International (FES-I), a Quality of Life Questionnaire (EQ-5D-5L), and the amount of moderately intensive physical activity (PA). To compare the falls during the years before and after the home visit, the mean number of falls before the visit was calculated based on reported falls during a) the year and b) the month (multiplied by 12) before the visit. A 2:1 weighted mean from a) and b) was calculated, considering that retrospective reporting over one year or over one month may underestimate or overestimate, respectively, the number of falls. Statistical analysis fitted a GEE-Poisson-Modell (Generalized Estimating Equations) to the data for number of falls and a Linear Mixed Model (LMM) for fear of falling, quality of life and PA. Falls related health care spending in the years before and after the intervention were compared based on health insurance claims data.

Results: Overall, 639 person-years of observation time were available for analysis. Participants were mainly female $(59 \%)$ and had a mean age of $81.8+/-5.2$ years. On average, rate of falling decreased from 1.35 to 1.02 per person year $(-23.9 \%)$, fear of falling decreased by $-1.27(95 \% \mathrm{Cl}-1.50,-1.05)$, quality of life improved by $-0.88(95 \% \mathrm{Cl}-1.09,-0.68)$, PA increased by 9.87 minutes per day $(95 \% \mathrm{Cl} 5.65,14.09)$. Health insurance claims data showed a $48.0 \%$ reduction $(95 \% \mathrm{Cl} 30.5 \%, 61.0 \%)$ of medically treated falls. The average cost per prevented medically treated fall was estimated at 1317 Swiss Francs (approximately 1200 Euro) and the probability of the intervention being cost-saving at $47 \%$.
Conclusion: This low-threshold fall prevention programme was found to be very effective and cost-effective. Home visits by trained PTs or OTs contribute substantially to reduce falls in the elderly. The high cost-effectiveness is explained by a substantial reduction of severe falls.

\section{REFERENCES:}

[1] Swiss Council for Accident Prevention BFU (2019); https://www.bfu.ch/de/ die-bfu/medien/statistik-der-nichtberufsunfaelle-1. Downloaded 15 Dec. 2020.

[2] Kempen $\mathrm{G}$ et al. The Short FES-I: a shortened version of the falls efficacy scale-international to assess fear of falling. Age and Ageing, 37(1)

[3] Herdman $M$ et al. Development and preliminary testing of the new five-level version of EQ-5D (EQ-5D-5L). Qual Life Res. 2011;20(10) 1727.

[4] Craig CL et al: International physical activity questionnaire: 12-country reliability and validity. Medicine and science in sports and exercise 2003, 35(8):1381-1395

Disclosure of Interests: None declared

DOI: 10.1136/annrheumdis-2021-eular.3453

\section{OP0159-HPR IMPACT OF NON-PHARMACOLOGICAL INTERVENTIONS TARGETING SLEEP DISTURBANCES OR DISORDERS IN PATIENTS WITH INFLAMMATORY ARTHRITIS: A SYSTEMATIC REVIEW AND META- ANALYSIS OF RANDOMISED TRIALS}

K. M. Latocha ${ }^{1}$, K. Løppenthin ${ }^{2,3}$, S. Al-Bazy ${ }^{1}$, T. Albrechtsen ${ }^{1}$, H. Jensen ${ }^{1}$ M. Østergaard ${ }^{1,3}$, P. Jennum ${ }^{3,4}$, B. A. Esbensen ${ }^{1,3}$, R. Christensen ${ }^{5,6}$. ${ }^{1}$ Rigshospitalet, Copenhagen Center for Arthritis Research, Center for Rheumatology and Spine Diseases, Glostrup, Denmark; ${ }^{2}$ Rigshospitalet, Research Unit for Cancer Late Effect, Department of Oncology, Copenhagen, Denmark; ${ }^{3}$ Copenhagen University, Department of Clinical Medicine, Faculty of Health and Medical Sciences, København, Denmark; ${ }^{4}$ Rigshospitalet, Danish Center for Sleep Medicine, Department of Clinical Neurophysiology, Glostrup, Denmark; ${ }^{5}$ The Parker Institute, Musculoskeletal Statistics Unit, Copenhagen, Denmark; ${ }^{6}$ University of Southern Denmark, Department of Clinical Research, Faculty of Health Sciences, Odense, Denmark

Background: Patients with inflammatory arthritis (IA) often present limitations in daily life due to unpredictability, severity and chronicity of the disease, reduced functional ability and health-related quality of life. Moreover, sleep disturbances or disorders have been reported in up to $40-90 \%$ of patients with IA [1-3]. Sleep disturbances are further associated with pain, fatigue, mental well-being and disease activity [4]. As chronic us of hypnotic drugs are associated with tolerance and side effects, there is growing interest in non-pharmacological management to improve sleep.

Objectives: With a systematic review and meta-analysis, our primary objective was to evaluate the evidence of non-pharmacological interventions targeting sleep in patients with IA. Primary outcome was sleep domains, while secondary objectives were based on the core outcome domains for IA trials and harms.

Methods: Following protocol closure, a systematic search was undertaken in MEDLINE, CENTRAL, PsycINFO, CINAHL, ClinicalTrials.gov, ACR and EULAR in September 2020. Eligible studies were randomised trials with adults with IA and concomitant sleep disturbances or disorders, comparing a non-pharmacological intervention targeting sleep disturbances or disorders to another non-pharmacological intervention, a pharmacological intervention or standard care. Screening of titles, abstracts, and subsequent full text assessment were conducted independently by two reviewers using the Covidence tool. Randomisation, blinding, and adequacy of analyses was assessed using the Cochrane's RoB tool and the overall quality of evidence was rated using GRADE methodology. Disagreements were resolved at consensus meetings with last authors Effect sizes for continuous outcomes were based on the standardised mean difference (SMD), combined using standard random-effects meta-analysis (al with $95 \%$ Cls).

Results: Six trials (308 patients) were included in the quantitative synthesis. Two trials included patients with the sleep disorder insomnia and the remaining four trials included patients with sleep disturbances. Sleep domains were measured with Pittsburgh Sleep Quality Index (PSQI) or Insomnia Severity index (ISI) Three trials reported improvement on sleep following foot reflexology, auricular plaster therapy, and exercise. The overall meta-analysis presented in the figure of forest plot for self-reported sleep suggests that non-pharmacological interven tions have a potentially large effect size of $-0.80(95 \% \mathrm{Cl},-1.33$ to -0.28$)$ on sleep. However, the quality of the evidence was assessed as corresponding to low, given that the body of the evidence was rated down twice, due to serious study limitations and inconsistency. 


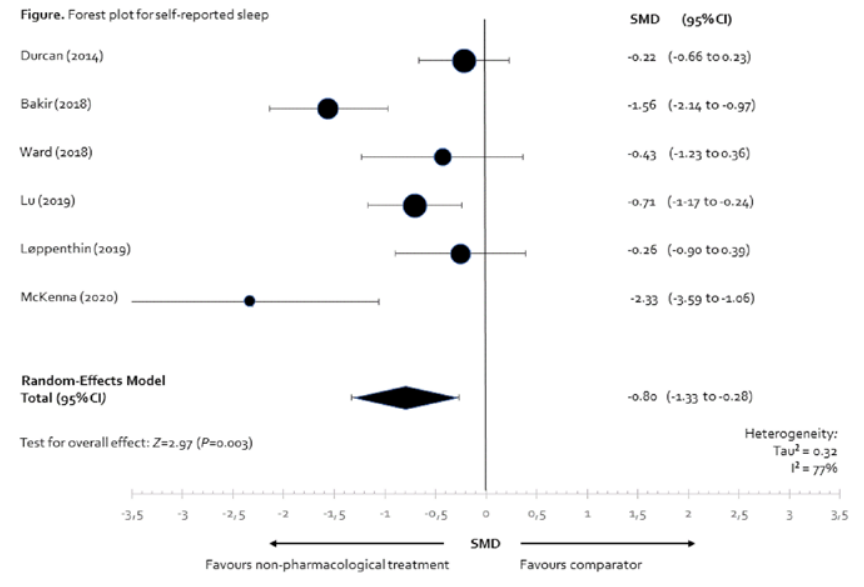

Conclusion: Although the effect of non-pharmacological interventions targeting sleep disturbances or the sleep disorder insomnia was statistically highly significant, the implication for clinical practice is questionable because of the overall quality evidence. None of the core outcomes used in contemporary IA trials have indicated clinical benefit in favour of non-pharmacological interventions targeting sleep disturbances or disorders.

In conclusion, more rigorous research on non-pharmacological management of sleep disturbances and disorders is urgently needed, also aimed at specific sleep disorders, in order to fully reveal the clinical utility of these novel treatment options. At this point, non-pharmacological treatment of sleep disturbances or disorders is promising and potentially highly effective, and may have the potential to persistently decrease the symptom burden and increase the quality of life of patients with IA.

REFERENCES:

[1] Li et al., Psychol Health Med. 2019 Sep;24(8):911-924

[2] Haugeberg et al., Arthritis Res Ther. 2020 Aug 26;22(1):198

[3] Wali et al., J Clin Sleep Med. 2020 Feb 15;16(2):259-265

[4] Løppenthin et al., Clin Rheumatol. 2015 Dec;34(12):2029-39

Disclosure of Interests: Kristine Marie Latocha: None declared, Katrine Løppenthin: None declared, Safa Al-Bazy: None declared, Tannie Albrechtsen: None declared, Helle Jensen: None declared, Mikkel Østergaard Speakers bureau: Abbvie, Celgene, Eli-Lilly, Janssen, Novartis, Pfizer, Roche, Sanofi and UCB, Consultant of: Abbvie, BMS, Boehringer-Ingelheim, Celgene, Eli-Lilly, Hospira, Janssen, Merck, Novartis, Novo, Orion, Pfizer, Regeneron, Roche, Sandoz, Sanofi and UCB, Grant/research support from: Abbvie, BMS, Merck, Celgene, Novartis, Poul Jennum: None declared, Bente Appel Esbensen: None declared, Robin Christensen: None declared

DOI: 10.1136/annrheumdis-2021-eular.836

\section{OP0160-HPR PREFERENCES FOR TREATMENTS TO PREVENT RHEUMATOID ARTHRITIS: DISCRETE CHOICE SURVEY OF GENERAL POPULATIONS IN UNITED KINGDOM, GERMANY, AND ROMANIA}

G. Simons ${ }^{1}$, J. Veldwijk2 ${ }^{2}$, R. DI Santostefano ${ }^{3}$, M. Englbrecht ${ }^{4}$, C. Radawski ${ }^{5}$, L. Valor ${ }^{6}, \mathrm{~K}$. Raza $^{7}$, M. Falahee ${ }^{7}{ }^{1}$ University of Birmingham, Rheumatology Research Group, Institute of Inflammation and Ageing, Birmingham, United Kingdom; ${ }^{2}$ Erasmus University Rotterdam, Erasmus School of Health Policy and Management, Rotterdam, Netherlands; ${ }^{3} J a n s s e n ~ R \& D$, Janssen R\&D, NJ, United States of America; ${ }^{4}$ Freelance data scientist, Freelance data scientist, Eckental, Germany; ${ }^{5}$ Eli Lilly \& Company, Eli Lilly \& Company, Indianapolis, United States of America; ${ }^{6}$ Friedrich-Alexander-University Erlangen, Department of Internal Medicine, Erlangen, Germany; ${ }^{7}$ University of Birmingham, Rheumatology Research Group, Institute of Inflammation and Ageing, Birmingham, United Kingdom

Background: There is increasing research focus on intervention for rheumatoid arthritis (RA) at the earliest stages of disease development, including treatment to prevent RA in at-risk groups. Novel cellular therapies are in development, and the effectiveness of existing immunomodulatory agents to prevent RA in those at risk is under investigation. Quantitative evidence of likely uptake of preventive treatments, and preferences for benefits and risks of such treatments is limited. Objectives: To quantify preferences for preventive therapies for RA.

Methods: A web-based survey $(n=2959)$ was administered to an age- and gender- stratified sample of adults in the general population from online survey panels in the UK, Germany, and Romania. After receiving information about RA, questions to check comprehension of background information, an introduction to the survey tasks and warm-up questions, participants were asked to imagine that they were experiencing arthralgia (without swelling) and had positive autoantibody tests indicating a $60 \%$ chance of developing RA in the next two years. Using a discrete choice experiment with a Bayesian D-efficient design, participants were offered a series of 15 choices between no treatment and two unlabeled hypothetical treatments to lower risk of RA development. Treatments were defined by six attributes with varying levels including benefits, risks, and frequency/route of administration (Table 1). One choice task with fixed levels described treatments representative of those under investigation for RA prevention (abatacept, hydroxychloroquine, atorvastatin and tolerogenic cell-based therapy). Attribute selection and presentation was informed by previous qualitative research, ranking surveys, systematic literature review, and expert opinion. Survey design was informed by patient research partners. The survey was pretested during qualitative interviews and revised. A pilot of the final survey with 100 respondents was conducted to obtain priors for the final experimental design. Random parameters logit (RPL) models were used to estimate relative importance of treatment attributes and likely treatment uptake rates in each country.

Table 1. Treatment attributes and levels

\begin{tabular}{ll}
\hline Attribute & \multicolumn{1}{c}{ Levels } \\
\hline $\begin{array}{ll}\text { Chance of developing RA reduced from } 60 \% \text { to } \\
\text { How the treatment is taken }\end{array}$ & $\begin{array}{l}10 \% ; 20 \% ; 30 \% ; 40 \% \\
\text { A shallow injection } \\
\text { under the skin }\end{array}$ \\
& $\begin{array}{l}\text { A drip into the vein } \\
\text { One or two tablets }\end{array}$ \\
How often the medication has to be taken & Daily \\
& Weekly \\
& Monthly \\
Chance of mild side effects & Every 6 months \\
Chance of a serious infection due to treatment & $2 \% ; 5 \% ; 10 \%$ \\
Chance of a serious side effect that is potentially irreversible & $0 \% ; 1 \% ; 5 \%$ \\
& 1 in 100,000 people \\
& 20 in 100,000 people \\
& 100 in 100,000 people
\end{tabular}

Results: Across all three countries, effectiveness was the treatment attribute that had most impact on treatment choice (Figure 1). Method of administration was second most important for respondents from the UK and Romania but less important for German respondents. Risks of serious infection and serious side effects were more important determinants of treatment choice for respondents in Romania than they were in the UK and Germany. Percentage choice of fixed profiles reflecting abatacept, atorvastatin, hydroxychloroquine, tolerogenic cellbased therapy and no treatment differed across countries $\left(\chi^{2}=78.90 ; p<0.001\right)$ : $28.3 \%, 20.6 \%, 22.2 \% 18.5 \%$ and $10.4 \%$ respectively in the UK; $31.3 \%, 18.8 \%$, $11.2 \%, 23.4 \%$ and $15.3 \%$ in Germany; and $27.6 \%, 20.5 \%, 15.8 \%, 21.7 \%$ and $14.4 \%$ in Romania.

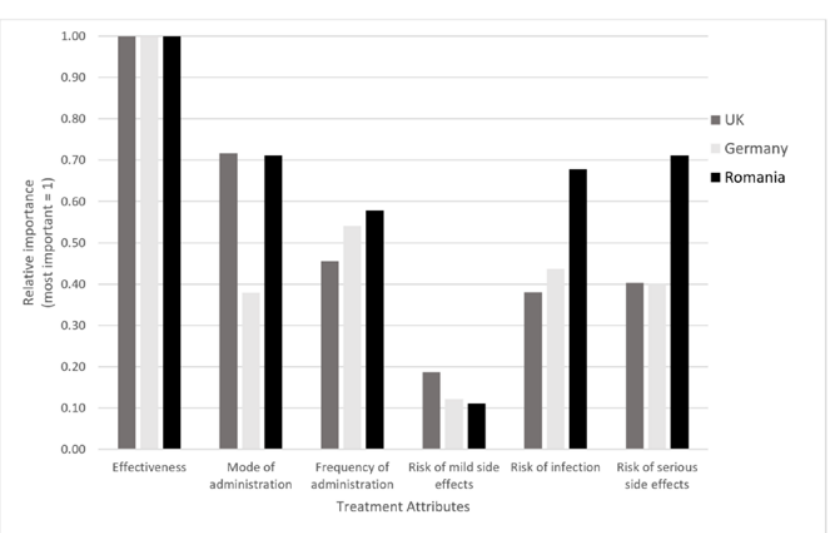

Figure 1: Relative importance of treatment attributes stratified by country; UK ( $N=982$ ), Germany $(N=984)$ and Romania $(N=993)$. Separate RPL models were used for each country.

Conclusion: This study suggests that effective preventive treatments for RA are acceptable to members of the general population told to assume up a $60 \%$ chance of developing RA. The relative importance of treatment attributes and likely uptake of fixed treatment profiles differed across countries. These findings are informative for the design of prevention trials, and the development of informational resources and efficient preventive strategies for those at risk of developing RA.

Acknowledgements: On behalf of the PREFER project. PREFER received funding from the IMI 2 Joint Undertaking (grant No. 115966), which receives support from the EU's Horizon 2020 research and innovation program and European Federation of Pharmaceutical Industries and Associations (EFPIA). K. Raza is supported by the NIHR Birmingham Biomedical Research Centre. 